\title{
A Rare Case of Epidermoid Carcinoma on Mandibular Osteonecrosis Due to Oral Bisphosphonates in the Treatment of Osteoporosis. Case Report and Review of Literature
}

\author{
Sánchez López $\mathrm{JD}^{1 *}$, Pérez de Perceval Tara $\mathbf{M}^{2}$, Cariati $\mathbf{P}^{2}$, Rodríguez Ismael ${ }^{3}$ \\ ${ }^{1}$ Oral and Maxillofacial Surgery Consultant. Complejo Hospitalario Universitario de Granada, \\ Spain. \\ ${ }^{2}$ Oral and Maxillofacial Surgery Resident. Complejo Hospitalario Universitario de Granada, Spain. \\ ${ }^{3}$ Dentist. Professor of Dentistry, University of Córdoba, Argentina.
}

Received: March 14, 2018; Accepted: March 28, 2018; Published: April 04, 2018

*Corresponding author: Sánchez López JoseDarío, Oral and Maxillofacial Surgery Consultant. Complejo Hospitalario Universitario de Granada, Spain. E-mail: josed.sanchez.sspa@juntadeandalucia.es

\section{Introduction}

Bisphosphonates (BP) are a group of synthetic analogs of inorganic pyrophosphate. They are used intravenously in the treatment of bone metastases of solid tumors and orally in the treatment of numerous rheumatic diseases, including osteoporosis [1].

Its mechanism of action is manifold, with recent published studies recognizing: inhibition of osteoclastic bone resorption, induction of osteoblasts to the secretion of an osteoclastmediated inhibition of bone formation, induction of apoptosis in osteoclast ("programmed cell death"), an antiangiogenic effect that reduces endothelial growth factor (EFG). Also, associated with the previous ones, there is an alteration of the macrophages and the cicatrization of the oral mucosa by involvement of the fibroblasts and keratinocytes [2].

Maxillary Osteonecrosis (BRONJ) associated with the use of intravenous BP in the treatment of bone metastases of solid tumors was first described by Ruggiero in 2004 [3]. The diagnostic classification of this entity was initially proposed in 2009 [4]. It has been recently modified in 2014, expanding to other anti-reabsorbing bone and antiangiogenic drugs [5].

Intraoral and oropharyngeal carcinomas have as their main risk factors alcohol, tobacco and human papillomavirus infection, especially the high risk oncogenic genotypes 16 and 18. All these factors may act separately or synergistically [6].

In the present study, we report a rare case of BRONJ induced by oral BP in the treatment of osteoporosis and its subsequent evolution towards squamous cell carcinoma.

Key words: Mandibular Osteonecrosis; Osteoporosis; Epidermoid Carcinoma

\section{Case Report}

Caucasian woman currently 56 years of age with no clinical records of interest (no diseases or toxic habits). She started treatment with chronic oral BP (Ibandronic Acid $2.5 \mathrm{mg} /$ day dose) in 2001 because of polyarthralgia and active osteoporosis. She was in high risk of developing pathological fractures according to densitometry studies.

In March 2012 she was assessed in our ward for presenting mobility, pain and haemorrhage at the 23 tooth with a vestibular table exposure for more than 6 months. First-stage orthopantomography (Figure 1) and subsequent facial CT scan (Figure 2) were performed, confirming the existence of type III

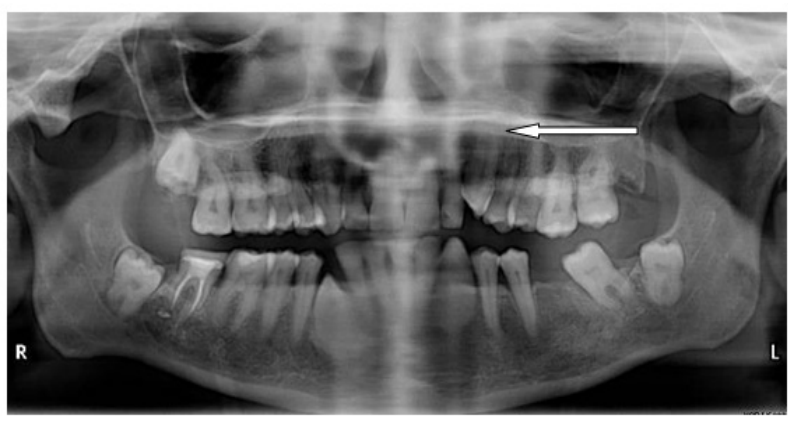

Figure 1: Orthopantomography showing well delimited osteochondensing are at tooth 23 level (White arrow).

BRONJ (according to the classification previously described). It was probably related to prolonged use of BP (more than 10 years). It was decided to practice surgical treatment of the lesion, after treatment for three days with broad-spectrum anti biotherapy. Under local anaesthesia an exodontia of the 23 tooth was performed, and an alveolar curettage with excision 
of bone sequestration and direct closure was done (Figure 3). Subsequently, antibiotic therapy was initiated according to the protocol of our Unit* based on recommendations from panel of experts and pain control (oral amoxicillin-clavulanic acid 875/125 mg, 3 times / day for 15 days, associated with rinses of $0.12 \%$ chlorhexidine, 2-3 times / day and stopping BP administration.

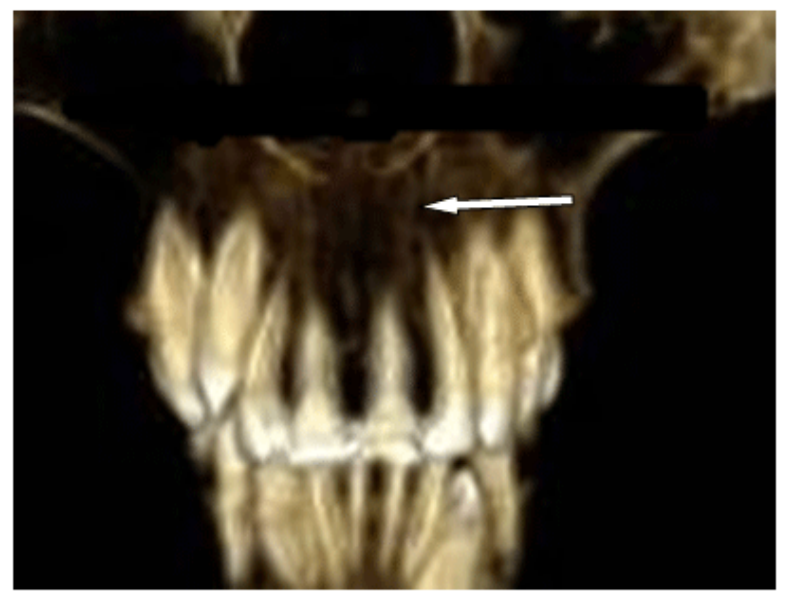

Figure 2: 3D CT scan compatible with osteonecrosis of maxillary tuberosity. Bone osteocondensation at tooth 23 level and a radiolucent area around tooth 22 was present. (White arrow).

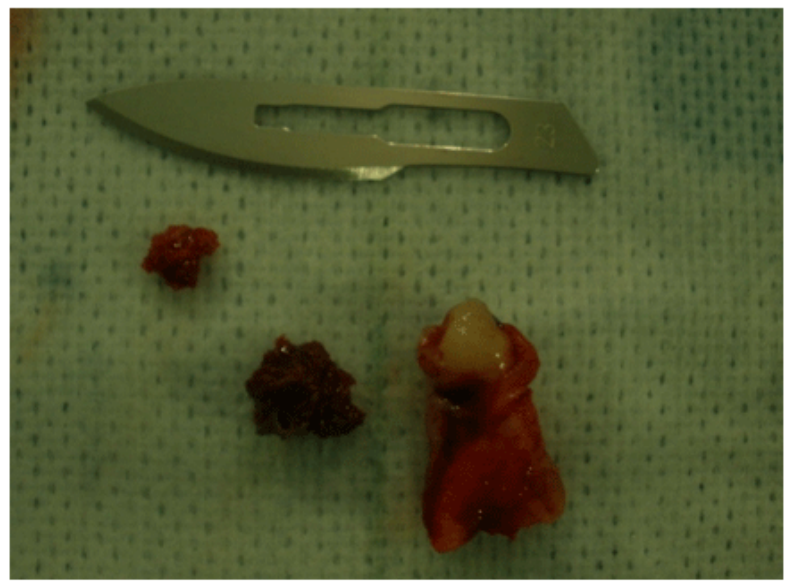

Figure 3: Surgical specimen showing tooth 23 surrounded by necrotic tissue and bone sequestration.

The patient outcome was highly favourable, with adequate cicatrization of the surgical wound. Only one month after the intervention, clinical examination showed soft tissue enlargement in the region treated, with pain and occasional bleeding. A control CT scan was performed showing left paramedical superior alveolar tuberosity osteolysis. A biopsy of the lesion was performed confirming the existence of moderately differentiated and infiltrating epidermoid carcinoma, classified as cT4aN0MxG2 (S IVa) (according to the TNM classification) **

On 6/5/2012 segmental hemimaxillectomy was performed under general anaesthesia. It confirmed squamous cell carcinoma of $0.5 \mathrm{~cm}$ with infiltration of bone trabeculae and anterior border invasion (pT4aN0MxR1 (SI)). Postoperative RT was administered. The patient remained in complete clinical remission until 7/8/2016, showing no tumor in imaging studies (Figure 4). PET-CT detected left submandibular hypermetabolic focus, and the decision to perform homolateral cervical dissection was made. After that, the presence of a metastatic adenopathy with nodal rupture was confirmed, initiating treatment with RT and chemotherapy.

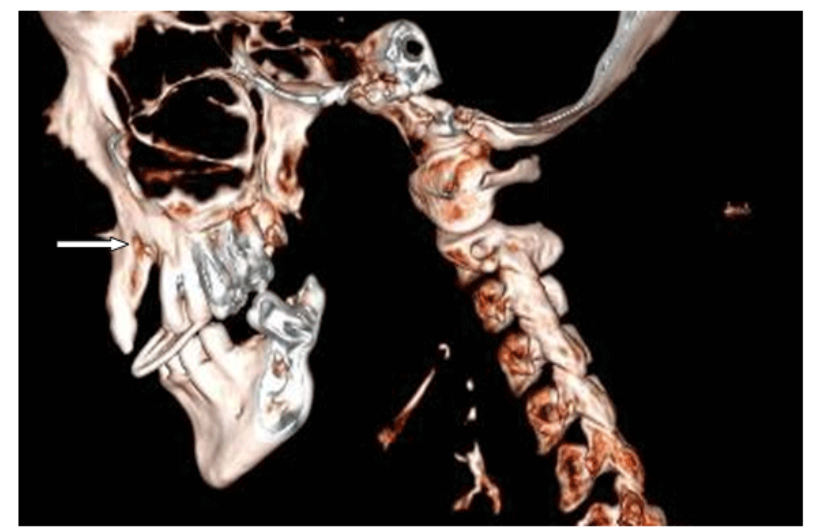

Figure 4: Postoperative control with 3D CT scan showing bone defect corresponding to segmental maxilectomy. (White arrow).

* Report of the Council of Scientific Affairs. Expert panel recommendations: Dental management of patients on oral bisphosphonate therapy 2006.

** The TNM classification system we include in this guide is based on the latest edition published by the UICC and the American Joint Committee on Cancer (AJCC) in January 2010.

\section{Discussion}

Despite the risk of induction of BRONJ, the use of oral BP in the treatment of osteoporosis is widespread, since its prevalence is low. In a study of 13000 patients, the risk of developing this pathology in long-term BP treatment was lower, with a prevalence of $0.1 \%$ ( 10 cases per 10,000 exposed), being estimated to increase to $0.21 \%$. More recently, other authors such as Malden and Lopez (2012) reduce this percentage to $0.004 \%$ ( 0.4 cases per 10000 exposed) in a population of 90000 patients in Southern Scotland [7].

The case report presented shows certain infrequent characteristics that we will discuss below. Firstly, the absence of risk factors that predispose to developing a BRONJ, such as poor oral hygiene or surgical manoeuvers (dental extractions) during treatment with BP and presentation in the upper jaw [8]. A proportion of jaw risk of 2:1 with respect to upper maxilla was proposed [9]. On the other hand, it emphasizes the intensity of the BRONJ in its first manifestation (SIII), which could be attributed to a prolonged use (over 10 years) with Ibandronic acid (nitrogen BP with a greater power than alendronate, the latter usually used in the treatment of osteoporosis). Moreover, Conte-Neto et al (2011) described two cases of BRONJ in women without risk factors with alendronate therapy for more than 3 years in the 


\section{Literature}

posterior part of the mandible [10]. This fact is explained because the half-life of BP in the bloodstream is very short, ranging from 30 minutes to 2 hours. Once absorbed by skeletal tissue it can persist for more than 10 years, which would explain its persistent long-term action on the bone.

The subsequent malignization of the osteonecrotic tissue in the context of BP uptake continues to be an exceptional and apparently paradoxical. BP, in consonance with different studies carried out in vitro, have antitumor properties through the inhibition of proliferation and invasion of tumor cells, inhibition of adhesion of tumor cells to bone tissue, induction of apoptosis in several malignant cell lines and inhibition of the proteolytic activity of matrix metalloproteinase's [11].

Though, these are experimental studies that require their clinical application.

In the bibliography consulted, we have only found the relationship between the use of BP and esophageal carcinoma [12]. Recently, authors like Gander report three cases of BRONJ with bone exposure underlying soft tissue of areas of malignization [13].

Conclusion is that BRONJ, irrespective of its clinical and radiological diagnosis, an anatomopathological study has to be performed in search of malignization areas.

\section{References}

1. Watts NB. Long-term risks of bisphosphonate therapy. Arq Bras Endocrinol Metabol 2014; 58(5):523-529.

2. Kramer J, Fantasia J. Bisphosphonates and Osteonecrosis of the jaws: review of Clinical Features and the Drug Effect on Oral Tissue. Clinic rev Bone Miner Metab 2011; 9:38-46.

3. Ruggiero SL, Mehrota B, Rosemberg TJ, Engroff SL. Osteonecrosis of the jaw associated with the use of biphosphonates. A review of 63 cases. J Oral Maxilofac Surg. 2004; 62(5):527-534.

4. Ruggiero SL, Dodson TB, Assael LA, Landesberg R, Marx RE, Mehrotra B. American Association of Oral and Maxillofacial Surgeons position paper on bisphosphonate-related osteonecrosis of the jaws-2009 Update. J Oral Maxillofac Surg. 2009; 67(5 Suppl):2-12. doi: 10.1016/j. joms.2009.01.009
5. Ruggiero SL, Dodson TB, Fantasia J, Goodday R, Aghaloo T, Mehrotra B, et al. American Association of Oral and Maxillofacial Surgeons. J Oral Maxillofac Surg. 2014; 72(10):1938-1956. doi: 10.1016/j. joms.2014.04.031

6. Scully C, Bagan J. Oral squamous cell carcinoma: overview of current understanding of aetiopathogenesis and clinical implications. Oral Dis. 2009; 15(6):388-399. doi: 10.1111/j.1601-0825.2009.01563.x

7. Malden N, Lopez V. An epidemiological study of alendronate related osteonecrosis of the jaws. A case series from the south-east of Scotland with attention given to case definition and prevalence. J Bone Miner Metab. 2012; 30(2):171-182. doi: 10.1007/s00774-011-0299-z

8. Hess LM, Jeter JM, Benham-Hutchins M, Alberts DS. Factors associated with osteonecrosis of the jaw among bisphosphonate users. Am J Med. 2008; 121(6):475-483.e3. doi: 10.1016/j.amjmed.2008.01.047

9. Hoff AO, Toth BB, Altundag K, V. Guarneri, A. Adamus, A. K. Nooka, et al. Osteonecrosis of the jaw in patients receiving intravenous bisphosphonate therapy. ASCO Annual Meeting Proceedings (postmeeting edition). J Clin Ocol. 2006; 24:8528. doi: 10.1200/ jco.2006.24.18_suppl.8528

10. Conte-Neto N, Bastos AS, Spolidorio LC, Marcantonio RA, Elcio Marcantonio. Oral biphosphonates-related osteonecrosis of the jaws in rheumatoid arthritis patients: a critical discussion and two case reports. Head Face Med 2011; 7:7. doi: 10.1186/1746-160X-7-7

11. Dedes PG, Gialeli Ch, Tsonis AI, Kanakis I, Theocharis AD, Kletsas D, et al. Expression of matrix macromolecules and functional properties of breast cancer cells are modulated by the bisphosphonate zoledronic acid.Biochim Biophys Acta. 2012; 1820(12):1926-1939. doi: 10.1016/j.bbagen.2012.07.013

12. Ho YF, Lin JT, Wu CY. Oral bisphosphonates and risk of esophageal cancer: a dose-intensity analysis in a nationwide population. Cancer Epidemiol Biomarkers Prev. 2012; 21(6):993-995. doi: 10.1158/1055-9965.EPI-12-0259

13. Gander T, Obwegeser JA, Zemann W, Grätz KW, Jacobsen C. Malignancy mimicking bisphosphonate-associated osteonecrosis of the jaw: a case series and literature review. Oral Surg Oral Med Oral Pathol Oral Radiol. 2014; 117(1):32-36. doi: 10.1016/j.oooo.2013.08.028 